\title{
Design of Gamma Spectrometer Based on NI software and Hardware Platform
}

\author{
Wei Jiang \\ Fundamental Science on Radioactive Geology and Exploration Technology Laboratory, East China \\ University of Technology, Nanchang, Jiangxi, China \\ jw186@163.com
}

Keywords: Gamma; LabVIEW ; Platform

\begin{abstract}
The gamma spectrometer is a radiometer that measures the gamma ray energy distribution and radiation dose. It is an important nuclear science physics method. It is one of the main methods to solve the problems of the earth sciences, environmental sciences and other issues. In this paper, virtual instrument technology is applied to the design of gamma spectrometer. The whole system consists of detectors, signal conditioning circuits, high-speed A/D acquisition cards, and upper-level performance analysis and processing software. It will detect gamma-ray conversion. As the trigger pulse, the pulse signal and the trigger signal are transmitted to the acquisition card at the same time, and the collected pulse data is transmitted to the computer for processing. The computer receives the data transmitted by the high-speed acquisition card to realize the detection, acquisition and analysis of the spectrum signal. The research, processing, storage, display, printing, retrieval and network transmission functions have important theoretical and practical values.
\end{abstract}

\section{Introduction}

The gamma spectrometer is a radiometer for measuring the gamma ray energy distribution and radiation dose. It is an important nuclear geophysical method and one of the main methods for solving problems related to earth sciences and environmental sciences. Based on the measured gamma ray energy spectrum, total gamma radiation, uranium, thorium, potassium, and other elements and other related elements and lithology information can be obtained. Based on this information, not only can uranium exploration be carried out, but it can also be applied to geological mapping, oil and gas exploration, the search for various metal and non-metallic minerals, and radiation monitoring of building materials and the environment. In a word, the application of gamma spectroscopy measurement and analysis technology is very extensive. It plays an important role in nuclear physics, nuclear energy utilization, nuclear protection, and environmental radiation analysis. In order to more accurately obtain the results of gamma spectrometry measurement and analysis, a multi-channel gamma spectrometer that studies how to acquire and process energy spectrum data is always a very important issue and is the direction of unremitting efforts[1-4].

However, the vast majority of gamma spectrometers currently use dedicated multi-channel analyzers. The entire system lacks sharing and flexibility, and the price is very expensive. In order to improve the interoperability and flexibility of the system and minimize the cost at the same time, based on a thorough investigation of the research status of instruments at home and abroad, the virtual instrument technology is used to design and develop a gamma spectrometer. It is not only possible to make full use of the abundant resources of computers, but also to be flexible, user-friendly, low-cost, and easy to expand and modify. This is the key to the topic selection and research significance of this project.

\section{System Hardware Design}

The gamma spectrometer based on the NI hardware and software platform mainly includes four parts: detectors, signal conditioning circuits, high-speed A/D acquisition cards, and upper level acquisition and analysis software. The detectors use $\mathrm{NaI}(\mathrm{Tl})$ crystals. Detection of gamma rays is converted into 
electrical pulses, output negative pulse voltage signal; signal conditioning circuit inverts the pulse signal, amplifies and forms a Gaussian-shaped signal, while forming a trigger pulse, the pulse signal and the trigger signal after finishing it are simultaneously passed to the acquisition card; after the acquisition card receives the trigger signal, it will begin to collect the pulse waveform, and pass the collected pulse data to the upper computer processing software; the computer software realizes the control of the high-speed acquisition card, and receives the high speed. Capture the data passed by the card and perform amplitude analysis on the data and map it to the appropriate channel. The software also performs a series of processing on the spectral data, calls the display unit, and displays the spectral lines. The software controls a series of parameters in the signal conditioning circuit based on the user's operation and its own analysis[5-6].

The system uses a $\mathrm{NaI}(\mathrm{Tl})$ crystal sensor as a detector. The detector consists of a $\mathrm{NaI}(\mathrm{Tl})$ scintillator, a photomultiplier tube, and a preamplifier circuit.

The main function of the signal conditioning circuit is to invert, sort, and amplify the probe pulses; and to generate trigger levels that are sent to the capture card. It is convenient for the acquisition card to perform short-term high-speed sampling after receiving the sampling instruction; at the same time, a DAC-controlled gain amplifier should be placed in the signal conditioning circuit to achieve programmable control of spectrum drift. The circuit adopts the DAC amplification circuit that is more commonly used at present: the dynamic signal that needs to be amplified is input to the reference end of the DAC, and the digital signal of the input is controlled to adjust the amplification factor of the output analog signal. If the input digital quantity is not changed, the amplification is performed. The multiple is fixed. If you need to change the magnification, you only need to change the input value of the digital terminal in the microprocessor program. Secondly, the signal conditioning circuit must also realize power supply regulation. Using a high voltage module, a high voltage is generated to power the photomultiplier tube in the sodium iodide probe. In order to ensure the safe operation of the high-voltage power supply, the power supply adjustment is expected to be manually adjusted using a sliding rheostat. The core part of the whole signal conditioning circuit needs to be controlled by the single-chip microcomputer and is mainly responsible for receiving the control instructions of the computer processing program, and realizes the adjustment of the DAC program-controlled amplifying unit in the circuit, and utilizes the A/D acquisition unit provided in the processor. The voltage of the high-voltage power supply is collected and displayed, which is convenient for the operator to observe and control intuitively.

Due to the difficulty in hardware implementation of high-speed A/D sampling circuits and the difficulty in the development of subsequent programs and drivers, this project chose to implement high-speed A/D full-peaks based on high-speed A/D acquisition cards already available on the market. For the information collection and understanding of the main kinds of $\mathrm{A} / \mathrm{D}$ sampling modules that already exist on the market, select the appropriate acquisition card with a qualified price. The main research contents are the design and completion of the signal conditioning hardware circuit with the data acquisition card, and the preparation of the data processing program. According to the characteristics of the gamma ray pulse, the rising edge of the pulse is about 2 microseconds to 3 microseconds. If you want to ensure the accuracy of the peak sampling, the number of data samples at the rising edge should be greater than 30 , so the acquisition card sampling The rate is at least greater than $15 \mathrm{M}$. According to this requirement, the use of NI's high-speed acquisition card PXI-6115 can meet the acquisition requirements.

\section{System Software Design}

The data acquisition program drives the acquisition card: parameters such as sampling mode and trigger mode are written into the acquisition card. After the acquisition card sends the completed acquisition instructions, the program reads the collected data from the onboard FIFO on the acquisition card. Filtering data entered into the program to make the waveform of the data smoother, eliminating the influence of partial track width unevenness. The program feeds the data into the amplitude analysis VI and analyzes the actual peak value of the useful pulse waveform based on the given threshold. Since the 
baseline in the circuit already has a dc component, the actual peak height needs to be processed and the dc voltage component existing in the circuit before the pulse enters the circuit is subtracted to obtain the net peak height of the pulse. After the net peak is scaled, the scale will be calibrated. The value is added to the corresponding one of the line arrays. After the processing is completed, the program waits for the next data read command. The program will call the drawing control to draw the spectral line at a certain time and perform real-time spectrum stabilization. When the program is calibrated, the DAC amplification unit in the drive signal conditioning circuit and the constant modification of the scale factor determine the peak positions of the peak and the potassium peak, and finally record the optimal magnification and scale factor.

The PC in the Window XP environment, with NI's graphical programming software LabVIEW as a development platform, through the data acquisition module to obtain energy spectrum measurement data, save the data and data processing analysis, to get gamma spectrum information. A modular program design method was adopted. The software was mainly composed of acquisition modules, data storage, data processing, and graphic display modules. According to the requirements of energy spectrum measurement, it was determined that the entire gamma spectrometer software mainly includes the following functional modules: acquisition control, data acquisition, multi-channel pulse amplitude analysis, spectrum analysis, and file management functions.

Finally, the use of NI's graphical programming software LabVIEW powerful network transmission capabilities to achieve the remote transmission of the signal, the dynamic data release and sharing, implementation methods include TCP technology, UDP technology, DS technology, PR technology, Web page publishing technology.

\section{Summary}

Most current gamma spectrometers use dedicated multichannel analyzers, which lacks sharing and flexibility and are expensive. In order to improve the interoperability and flexibility of the system and minimize the cost, the project is based on the NI software and hardware platform for the collection of gamma spectrum data, and the virtual instrument technology is applied to the design of the gamma spectrometer. The entire system consists of detectors, signal conditioning circuits, high-speed A/D acquisition cards, and upper level analysis and processing software. The detector uses NaI crystals to convert gamma rays into electrical pulses and outputs negative pulse voltage signals. The signal conditioning circuit inverts the pulse signal, amplifies and forms a Gaussian shaped signal, forms a trigger pulse, and simultaneously transmits the pulse signal and the trigger signal to the acquisition card; the acquisition card collects the pulse waveform after receiving the trigger signal; Pass the collected pulse data to the computer for processing; The computer software realizes the control of the high-speed acquisition card, receives the data transmitted by the high-speed acquisition card, and analyzes the data to realize the detection, acquisition and analysis of the spectrum signal, processing, storage, display, printing, retrieval and network transmission.

\section{Acknowledgements}

The technology research project of Jiangxi education department (GJJ171509) (GJJ171510) and the open project program of Jiangxi engineering research center of process and equipment for new energy (JXNE2017-07) and 2017 Fuzhou science and technology plan project and Educational reform project in Jiangxi province (JXJG-17-37-3) and 2014 Jiangxi university students innovation and entrepreneurship project supported this work.

\section{References}

[1] S.D.Schery. Thoron in the environment. Air \& waste management association. 40(1990) 493-497.

[2] F.Yuan,Y.Z.Li.Design of gamma energy spectrometer based on the technology of LabVIEW and bluetooth . Advanced Materials Research, 113 (2010) 720-724. 
[3] S.J.Cai Design and Test of Virtual Gamma-ray spectrometer. Automation \& Instrumentation, 24 (2011) 426-432.

[4] J.X. Zhang, J. Zhang, A portable natural gamma spectrometer with a special spectrum-stabilization technique.Nuclear Techniques, 28(2005)638-640.

[5] W.C. La, L.Q. Ge, A new portable multi-channel X spectrometry based on inserted PC104 computer . Nuclear Electronics \& Detection Technology, 24 (2004)37-39.

[6] S.Q. Zheng, W.D. Huang, Development of gamma-ray energy spectrum remote-measurement system based on LabVIEW. Nuclear Techniques, 29(2006)549-555. 